\title{
Experimental Models of Maternal Obesity and Neuroendocrine Programming of Metabolic Disorders in Offspring
}

\author{
Clare M. Reynolds, Stephanie A. Segovia and Mark H. Vickers*
}

Liggins Institute, University of Auckland, Auckland, New Zealand

Evidence from epidemiological, clinical, and experimental studies have clearly shown that disease risk in later life is increased following a poor early life environment, a process preferentially termed developmental programming. In particular, this work clearly highlights the importance of the nutritional environment during early development with

OPEN ACCESS

Edited by: Susan Ozanne, University of Cambridge, United Kingdom

Reviewed by: Laura Dearden, University of Cambridge, United Kingdom Paul D. Taylor, King's College London, United Kingdom

Thomas Prates Ong,

University of São Paulo, Brazil

*Correspondence: Mark H. Vickers m.vickers@auckland.ac.nz

Specialty section: This article was submitted to Neuroendocrine Science, a section of the journal Frontiers in Endocrinology

Received: 31 March 2017 Accepted: 06 September 2017 Published: 25 September 2017

Citation: Reynolds CM, Segovia SA and Vickers MH (2017) Experimental Models of Maternal Obesity and Neuroendocrine Programming of Metabolic Disorders in Offspring.

Front. Endocrinol. 8:245. doi: 10.3389/fendo.2017.00245 alterations in maternal nutrition, including both under- and overnutrition, increasing the risk for a range of cardiometabolic and neurobehavioral disorders in adult offspring characterized by both adipokine resistance and obesity. Although the mechanistic basis for such developmental programming is not yet fully defined, a common feature derived from experimental animal models is that of alterations in the wiring of the neuroendocrine pathways that control energy balance and appetite regulation during early stages of developmental plasticity. The adipokine leptin has also received significant attention with clear experimental evidence that normal regulation of leptin levels during the early life period is critical for the normal development of tissues and related signaling pathways that are involved in metabolic and cardiovascular homeostasis. There is also increasing evidence that alterations in the epigenome and other underlying mechanisms including an altered gut-brain axis may contribute to lasting cardiometabolic dysfunction in offspring. Ongoing studies that further define the mechanisms between these associations will allow for identification of early risk markers and implementation of strategies around interventions that will have obvious beneficial implications in breaking a programmed transgenerational cycle of metabolic disorders.

Keywords: developmental programming, maternal nutrition, neuroendocrine, appetite regulation, epigenetics, metabolic syndrome

\section{INTRODUCTION}

Evidence from clinical and experimental studies has linked a maternal obesogenic environment to increase in the risk for the development of obesity and related cardiometabolic disorders in offspring in adulthood. As such, although there is a genetic component to obesity risk, environmental influences and gene-environment interactions are seen as primary mediators in the etiology of the current obesity epidemic $(1,2)$. Many of the perinatal exposures examined experimentally are associated with reorganization of neurodevelopmental pathways that play a role in energy expenditure and appetite regulation in ways that increase the later risk for obesity and related metabolic dysfunction 
in offspring (3). Although a consensus has been reached that an obesogenic environment in the periconceptual, pregnancy and lactation periods increases the risk of metabolic disorders in adulthood, the potential mechanisms that confer this increased risk are less well defined (4). One of the most investigated (and experimentally consistent) mechanisms is around that of altered development of the hypothalamus during critical periods of developmental plasticity. Evidence from a range of experimental model species has now shown that changes in the maternal diet can lead to alterations in the wiring of the neuroendocrine pathways in the hypothalamus that control energy balance and appetite regulation in offspring. Further, a number of key hormonal factors have been identified that may mediate early developmental malprogramming by maternal overnutrition including insulin, leptin and ghrelin. In this context, maternal obesity can modify blood-brain barrier (BBB) functionality with altered organization and permeability to such factors directed toward the arcuate nucleus of the mediobasal hypothalamus (ARH) (5). Obesity is a common characteristic observed in offspring born to high-fat diet (HFD)-fed mothers concomitant with hyperphagia that reflects altered hypothalamic development and related changes in the expression of these key neuropeptides (6). Both leptin (7-9) and insulin (10-12) are strongly neurotrophic, so alterations in availability of either during the period of developmental plasticity can underpin some of these adverse developmental changes. Under normal conditions, when adipose stores increase, leptin and insulin levels increase with activation of proopiomelanocortin (POMC) neurons and subsequent inhibition of neuropeptide $\mathrm{Y}(\mathrm{NPY})$ and agouti-related peptide (AgRP), thus providing a negative feedback loop that regulates intake and prevents obesity. This feedback system is perturbed following adverse developmental programming [i.e., the developmental origins of health and disease $(\mathrm{DOHaD})$ ] with alterations in ARC wiring appearing to be relatively conserved across model species. There is also a growing body of evidence that suggests a critical role for neuroimmune interactions during the prenatal period in the programming of neurobehavioral disorders in postnatal life (13).

Animal models allow controlled investigation of the interactions between genetics and early development and subsequent effects of early life perturbations on the programming of neural pathways which control the regulation of energy balance. A primary focus of early DOHaD experimental work was via models that utilized a range of maternal nutritional deficiencies (global or macronutrient imbalance including low protein diets) to induce fetal growth restriction. However, given the current obesogenic environment, there has been a move toward models that investigate the impact of maternal and/or neonatal overnutrition on programming of alterations in energy balance and appetite regulation in offspring. As with the maternal undernutrition models, a number of studies across a number of species and different overnutrition exposures have now similarly shown that an early life obesogenic environment leads to altered development of the central pathways that regulate appetite control and energy balance. Whether the mechanisms are the same remains to be elucidated but maternal intakes at both ends of the dietary spectrum are characterized by offspring obesity and metabolic disorders. This review will cover the range of experimental models of maternal obesity currently used, the nature of the early life exposures examined, potential commonalities in mechanisms and potential limitations of some of the approaches used.

\section{EXPERIMENTAL MODELS OF MATERNAL OBESITY}

A number of experimental models of maternal obesity have been developed across a range of model species, dietary compositions, and duration of exposures. Programmed outcomes are relatively conserved across the models used and include impaired insulin sensitivity, hypertension and endothelial dysfunction, increased adiposity (with hyperleptinemia), and altered appetite regulation (e.g., hyperphagia) (6). Overlaid on this are sex-specific effects, which to date have not been well characterized, and long-term transgenerational impacts (14).

Logistical considerations can be a primary driver for the choice of animal model and therefore rodents are the most commonly used due to short gestational length, short timeframe to maturity of offspring and the opportunity to examine transgenerational effects and sexually dimorphic responses to programming stimuli. Rodents also have the benefit of being easily manipulated genetically and use of targeted knockouts, for example, to elucidate mechanisms. Use of models in larger animals including the sheep, pig, or non-human primate (NHP), although potentially having the better translatability to the human condition due to developmental timing (and being particularly conducive to fetal studies), have limitations around cost, gestational length and time to offspring maturity.

\section{Rodents}

Most work to date has been undertaken in the rodent, primarily the rat. As above, although the rodent has some distinct logistical and cost advantages over other model species (short gestation length/lactation period, utility in transgenerational studies, and relative ease in manipulating genetically), the critical developmental windows in early life where the pathways that regulate energy balance and appetite control are set, differ significantly between humans and rodents (15). In the rodent, the development of neuronal pathways occurs early in the neonatal period while humans and other large model species these pathways develop in fetal life (16). Despite these differences in developmental windows, outcomes from rodent studies closely parallel those observations made in maternal obesity models in larger animals including sheep, swine, and NHP.

During early postnatal development in the rodent, axon outgrowth from ARH neurons is stimulated by leptin, and preautonomic NPY/AgRP/gamma-aminobutyric acid neurons are particularly sensitive to the known neurotrophic effects of leptin (17). Early work by Bouret et al. highlighted the trophic role of neonatal leptin in mediating the process of neurite outgrowth that eventually form the projections from the ARH to the paraventricular nucleus (PVN) and other areas of the hypothalamus (7). This window of action was specific to the neonatal period; neonatal leptin administration to leptin deficient $o b / o b$ mice normalized wiring of the $\mathrm{ARH}$, whereas treatment in the 
postweaning period had no effect. A number of studies have now similarly shown that maintenance of a "critical" leptin threshold during the early life period is required to facilitate normal tissue development and signaling pathways that are involved in metabolic homeostasis. In the rat, maternal obesity can lead to a period of neonatal hypoleptinemia and hypoinsulinemia although adulthood in these animals is characterized by obesity and insulin/ leptin resistance (18). This may be due to abnormalities in the neonatal leptin surge as regards both amplitude and timing with work by Kirk et al. showing that rat neonates born to obese mothers later display an abnormal and prolonged neonatal leptin surge (19). It also has been recently shown in mice that neonatal insulin action leads to impaired hypothalamic neurocircuit formation in response to a maternal HFD (20). Targeted abrogation of insulin signaling specific to POMC neurons of offspring of HFD fed mothers restored POMC innervation of preautonomic PVH neurons and corrected the impaired glucose tolerance otherwise seen in these animals.

It has been shown that maternal and postnatal overnutrition (induced via a reduction in litter size) can differentially impact on appetite regulation and energy metabolism (21). Maternal obesity has been linked to hyperlipidemia in offspring paralleled by increased hypothalamic POMC mRNA and reduced NPY expression; these changes being amplified in offspring in the presence of a postnatal obesogenic environment $(21,22)$. In these studies, maternal obesity induced via a cafeteria-style HFD, lead to alterations in the central appetite circuitry and thus promotion of early onset obesity. Neonatal overnutrition by reducing litter size compounded the effects of maternal obesity via alterations in peripheral lipid and glucose metabolism that exacerbated the metabolic dysregulation observed in offspring (21). It has also been shown that maternal obesity interacts with a postweaning HFD to further amplify the adult programmed phenotype characterized by hyperphagia, adiposity, and metabolic dysregulation and was linked to increased hypothalamic NPY signaling and leptin resistance (23).

Of note, hypothalamic reductions in NPY expression and increased POMC expression can be induced by both maternal and neonatal overnutrition (21) with the greatest changes seen in those offspring of obese mothers who are then exposed to neonatal overnutrition via litter size reduction. Moreover, reduced hypothalamic NPY and AgRP expression shown in pups raised in small litters has been proposed to be an adaptation which overcomes the effects of neonatal overnutrition in early life (23).

A maternal HFD can stimulate proliferation of neuroepithelial and neuronal precursor cells in the hypothalamus during the embryonic period (24). This subsequently leads to a stimulation in the differentiation and proliferation of neurons and subsequent migration toward areas of the hypothalamus where consequently there is a domination of newly formed neurones that express orexigenic peptides (24). This increased neurogenesis, concomitant with hyperlipidemia, may therefore result in the longer term physiological and behavioral changes observed in offspring following weaning, including hyperphagia, preference for dietary fat, and increased body weights.

In addition to compromising the BBB (5), maternal obesity during pregnancy in the mouse can stimulate proliferation and thereby numbers of astrocytes in the fetal and early neonatal hypothalamus (particularly the ARH), which appears to be mediated, during fetal life, by interleukin (IL)-6 (25). Maternal obesity in the mouse can also lead to persistent synaptic impairments in offspring during development of the neuronal circuitry and may arise due to increased oxidative stress during the lactation period $(26,27)$. Leptin receptors are also expressed in mouse hypothalamic astrocytes with conditional deletion leading to changes in glial cell morphology and synaptic inputs onto those neurons involved in appetite regulation (28). Maternal obesity alters energy sensors (including mTOR and pAMPK) and epigenetic responses which modulate gene expression and neuronal differentiation and therefore can contribute to the obesity and related metabolic disorders, including hyperphagia, observed in offspring (29). Hyperleptinemia in the preweaning period arising from maternal obesity can induce lasting changes in the central melanocortin system (30) and melanocortin-4 receptor in the $\mathrm{PVH}$ appears to be a mechanism underpinning the programming of hypertension in later life (30).

There is a large variation in the composition of the experimental diets used across the range of rodent models and this has been of some debate over the robustness of the approaches used (e.g., the single fat source diets versus the "cafeteria" or "junk food" style diets) (31). In the rat, a maternal "junk food" diet can alter food intake preferences in offspring concomitant with programmed alterations in the mesolimbic (reward) pathways (32). Alterations in the fat:carbohydrate balance also suggest that even minor changes in macronutrient balance may alter phenotype outcome. As an example, when a high carbohydrate/HFD is utilized, insulin hypersecretion may induce compensatory mechanisms that may not manifest when HF/low-carbohydrate diets are used (33).

Evidence from existing models suggest that a maternal obesogenic environment facilitates central orexigenic pathways to induce obesity in offspring, while neonatal overnutrition via a reduction in litter size appears to be more closely associated with anorexigenic adaptations, possibly as a measure to counteract overnutrition (23). Further, there are compounding detrimental effects arising due to interactions between maternal obesity, neonatal overnutrition and postweaning HFD intake that amplify the metabolic disorders observed in offspring. Of note, some of the differences that have been observed across maternal obesity studies in which relate to key hypothalamic markers may relate to fed-fasting conditions at the time of analysis (i.e., differential responses to fed/fasted states) and differences in the dietary paradigm used, i.e., cafeteria versus single-source fat HFDs and length of dietary exposure(s). In the fed state, a reduction in NPY and increases POMC mRNA are observed in offspring from obese mothers (34). Under fasting conditions, the downregulation in NPY expression can be normalized to that of the controls in the offspring of obese mothers. Therefore, in a fasted state, NPY was not shown to be different in HFD-fed animals (34). As regards dietary composition, no direct comparisons have been made in maternal obesity models but it has been shown in non-pregnancy models that different obesogenic diets can elicit differential effects on ARC leptin receptor, NPY, and AgRP expression (35). 


\section{Sheep}

It is well established that the leptin peak present in the rodent during the early neonatal period parallels the normal development of central appetite regulatory regions, and alterations in the profile of the so-called "leptin surge" predisposes to obesity and metabolic dysfunction in offspring in later life. However, unlike humans and larger animal models including the sheep, rodents are born at an immature (altricial) stage of development so work undertaken in newborn lambs allows determination of the potential relevance to human development (36). Adult-like localization of leptin receptor $(\mathrm{OB}-\mathrm{Rb})$ gene expression and primary appetite regulatory neuropeptides in the hypothalamus are established prior to birth in the sheep. Thus, central appetite regulatory neural networks that have the potential to respond to changes in maternal nutrient supply are already present in the sheep fetus, which may impact on regulation of energy homeostasis both before and after birth (37). Long et al. have shown that maternal obesity can lead to a diminished neonatal lamb plasma leptin peak concomitant with increased plasma cortisol levels. It has also been shown that a maternal obesogenic environment is linked to increased fetal adiposity and altered expression of key enzymes involved in mediating fatty acid biosynthesis in adipose tissue (38). Similar results have been reported by Nicholas et al. whereby oocyte/embryo exposure to maternal obesity had lasting consequences for lipid metabolism in offspring (39). Recent work has also reported that maternal obesity leads to hyperphagia resulting in adiposity associated with adipocyte hypertrophy and increased fatty acid synthesis in adult male offspring of overnourished mothers although no potential central regulatory mechanisms were investigated (40). In the sheep, inducing maternal hyperglycemia or overfeeding leads to hyperinsulinemia, increase adiposity and increases in fetal POMC gene expression. These effects appear to be specific to the POMC system with AgRP, NPY, and cocaine- and amphetamine-regulated transcript peptide in the ARH unaffected by either exposure although the longer term effects on offspring phenotype are not yet known (41-43).

\section{Pig}

Although a number of models of altered maternal nutrition have been established in the pig, there are little data on neuroendocrine readouts in the setting of maternal obesity. It has been shown that relative maternal undernutrition leads to piglets with an abnormal hypothalamic distribution of leptin receptors that is linked to altered food-intake behavior (44). This phenotype can be partially rescued via neonatal leptin administration. Maternal intake of a Western-style diet during pregnancy/lactation led to changes in microbiota profile, blood lipids, cognitive responses, and hippocampal neurogenesis in swine offspring (45). Recent work by Sanguinetti et al. has also shown that maternal HF feeding results in marked alterations in brain glucose metabolism in offspring thus predisposing to metabolic-neurodegenerative diseases (46-48).

\section{Rabbit}

In the rabbit, as reported for other model species, offspring from mothers consuming a HFD are characterized by an adverse cardiovascular profile in later life which appears to manifest as a consequence of an altered hypothalamic sensitivity to leptin and ghrelin $(47,48)$. Offspring of HFD-fed rabbits displayed resistance to the anorexic effects of central leptin administration, less neuronal activation in the ARH, and PVN in HFD offspring compared with rabbits fed a maternal control diet. A HFD in the rabbit has also been shown to alter leptin and the melanocortin signaling pathway in the ventromedial hypothalamus (VMH) of offspring (49). This model was characterized by obesity-related hypertension which could be normalized following leptin receptor antagonist injections to the $\mathrm{VMH}$.

\section{Non-Human Primate}

Less work on the neuroendocrine programming of energy balance has been undertaken in the NHP. Maternal consumption of a HFD leads to increased anxiety-like behavior and perturbations in the serotonergic system in NHP offspring (50). Independent of maternal obesity, intake of a HFD during pregnancy alone can result in a widespread activation of proinflammatory cytokines (including IL-1 $\beta$ and IL-1 type 1 receptor) that may lead to changes in the melanocortin system. Abnormalities in POMC expression in the fetus, if persisting postnatally, can impact upon a number of peripheral systems, including body weight regulation, cardiovascular function, and altered stress responsiveness (51). Of note, dietary recuperation of obese NHP mothers to a standard healthy gestational diet, resulted in normalization of fetal melanocortin levels. Rivera et al. have also recently shown that both maternal HFD consumption and maternal obesity in the NHP during early life results in offspring that had a higher risk of becoming obese as a consequence of increased intake of palatable energy-dense food, a behavior that was associated with a reduction in central dopamine signaling (52).

\section{GUT-BRAIN AXIS}

Appetite can be controlled by food intake-regulatory peptides that are secreted from the gastrointestinal tract including ghrelin, glucagon-like peptide 1, peptide YY, cholecystokinin, and the recently discovered nesfatin-1 via the gut-brain axis (53). As key hypothalamic centers regulate appetite and body weight in response to hormonal and other central stimuli, dysregulation of gut-brain communication may underlie programmed metabolic disorders, including obesity.

The "hunger hormone" ghrelin is an appetite-regulating factor, primarily produced in the stomach, and is involved in regulation of energy homeostasis via actions in the ARH including effects on NPY and AgRP neurons. The hypothalamic ghrelin-sensitive circuits are dynamically regulated by central insulin and leptin concentrations (54). Ghrelin has been shown to mediate neural fiber growth in the ARH during the neonatal period and, in the neonatal mouse, alterations in ghrelin action during this critical developmental period may lead to increased adiposity in adulthood (55). Ghrelin antagonism results in enhanced ARH neural projections and lasting metabolic effects that include impaired leptin sensitivity and an increase in body weight, central adiposity, and blood glucose concentrations. In non-pregnant rats, work by Schele et al. has shown that central administration of ghrelin 
can influence food choice and, in accordance with its role as a gut-brain hunger hormone, appears able to alter food choice in an acute manner, with notable effects on promotion of a more "healthy" chow intake. The ventral tegmental area was proposed as a neurobiological substrate that likely accounted for these observed effects (56). Further work by Briggs et al. has shown that diet-induced obesity leads to ghrelin resistance as a consequence of reduced NPY/AgRP responsiveness to circulating ghrelin. This leads to suppression of the neuroendocrine ghrelin axis in order to limit further food intake (57). Altered ghrelin in the setting of maternal obesity has yet to be well defined although in the setting of neonatal overnutrition ( $v i$ litter size reduction), pups display an impaired central response to peripheral ghrelin (58). Normalization of early hypoghrelinemia was relatively ineffective at correcting metabolic outcomes, suggesting ghrelin resistance in offspring raised in small litters.

Although the role of ghrelin has been well described broadly as a regulator of hypothalamic feeding pathways, little has been done as regards the potential differential effects of the two circulating forms of ghrelin; acylated versus des-acylated. Most research to date has focused on acyl ghrelin and the potential role of neonatal des-acyl ghrelin in developmental programming is not well defined. Recent work by Sominsky et al. has reported that early life overnutrition acutely affects ghrelin regulation in the short term, leading to a reduction in circulating des-acyl ghrelin and increased ARH expression of the growth hormone secretagogue receptor (59). These changes were paralleled by increases in neuronal activation in response to exogenous acyl, but not des-acyl, ghrelin in the ARH and PVN. Importantly, however, the observed effects on the ghrelin system arising from neonatal overnutrition did not persist into adulthood in the male offspring studied. Of note, these effects may be sexually dimorphic in nature as the same group has also recently reported that neonatal overnutrition leads to a persistent disruption of pituitary ghrelin signaling in females, potentially via exacerbating central stress responsiveness in these animals (60).

\section{EPIGENETICS AND THE REWARD PATHWAYS}

Obesity and related metabolic disorders reflect a complex interplay of multiple factors, with interactions across numerous different genetic and environmental factors with modern obesogenic environments exacerbating the genetic risk for obesity. Fixed genomic variation appears to explain only a small proportion of obesity risk (61). As such, epigenetic modifications [including altered DNA methylation, changes in histone modifications and microRNAs (miRNAs)] offer alternative mechanisms by which alterations in the nutritional environment in early life may exert lasting phenotypic consequences in offspring.

Although a number of studies have investigated changes in epigenetic marks in the setting of maternal undernutrition, particularly around methylation of the leptin and POMC promoter, less has been described for maternal obesogenic models. Moreover, most studies to date have examined effects in peripheral tissues with a relative paucity of data in these models as relates to changes in neuroendocrine pathways. As an example, overnutrition during lactation can result in epigenetic modifications in key genes that are known to be involved in the insulin signaling pathway in skeletal muscle that can manifest as impaired insulin sensitivity in later life (62). Neuroendocrine alterations have also been reported in this model as pertains to insulin and leptin sensitivity with changes in hypothalamic insulin receptor and POMC gene methylation status $(63,64)$ and thus offspring phenotype is likely a consequence of epigenetic changes across processes that represent a complex and highly integrated network across several tissue systems. Hypermethylation of the POMC promoter persists in offspring of HFD dams, even when offspring are recuperated onto a standard chow diet postweaning. This places them at an increased risk for hyperphagia when challenged with a HF diet (65). These data also showed POMC promoter demethylation in mothers fed a HFD during pregnancy and lactation concomitant with a heightened decrease in body weight during lactation. A maternal HFD leads to hepatic cell cycle inhibition via alterations in cyclin-dependent kinase inhibitor P21 (Cdkn1a) activity and associated changes in gene expression and DNA methylation in neonatal rat offspring (66). However, cell cycle changes as relates to central processes were not investigated but are also likely perturbed given the potential role for P21 as a modulator of neurosecretory activity of hypothalamic neurons (67) and changes in neuronal secretion of P21 following alterations in food availability (68) and inflammation (69).

Work in NHPs has shown that, in addition to alterations in DNA methylation profiles, an energy-dense maternal diet can alter chromatin structure in the fetus via covalent modifications of histones (70). Further, a maternal HFD alters neonatal hepatic metabolism, albeit in a sex-specific manner, and in association with histone modifications, may contribute to the known sexual dimorphic responsiveness as regards changes in oxidative balance (71). Fetal sirtuin 1 (SIRT1) histone and protein deacetylase activity in NHPs can be modulated by a maternal HFD and suggests a role for SIRT1 in the mediation of the fetal epigenome/ metabolome $(72,73)$. However, most studies have focused on hepatic readouts and whether similar histone changes occur in the neuroendocrine compartment has not been investigated but, as shown by Desai et al., are also likely to be perturbed with persistent changes in histone deacetylases observed in the ARC of offspring of obese mothers (29). miRNAs can also lead to histone modifications and changes in DNA methylation of promoters, which in turn affects the expression of target genes (74). In a sheep model of maternal obesity, miRNA expression in fetal muscle is altered (downregulation of miRNA let-7g expression) and therefore may represent a possible mechanism underlying the enhanced adipogenesis observed during development of fetal muscle (75). Whether there is a neuroendocrine contribution to this phenotype was not evaluated; given that the Let-7 family is highly expressed in the hypothalamus and proposed to be positively correlated with energy balance, further work is warranted as the central function of the Let-7 axis remains unclear (76).

In addition to homeostatic systems responsive to nutrient sensing and regulation of energy balance, maternal obesity can also perturb the hedonic system which includes stimuli related to reward and cognitive factors (4). In the context of early life 
developmental programming, exposure to HF/high-sugar diets has been shown to result in altered development of central reward systems, leading to increases in fat intake and altered responsiveness of the hedonic reward system to over consumption of energydense food in offspring in later life (32). As such, a maternal HFD can lead to altered gene expression and methylation of opioid and dopamine-related genes and thus may provide a further potential mechanism for programming of appetite control in offspring and a preference toward fatty/energy dense food intakes $(70,77)$. Obesity in mice at the time of conception itself has also been shown to program the opioid system in the offspring brain (77). Importantly for translative purposes, the reward pathways develops in utero in rodents with functional innervation in place by birth (78); investigation of the reward system may be therefore more translatable from rodent to the human than for some other processes (6).

Rats fed a "cafeteria-style" diet for even short periods of time display an impairment in sensory-specific satiety and these deficits persist even following dietary recuperation and withdrawal of the cafeteria-style diet (79). Further, feeding a cafeteria-style diet results in alterations in neuroadaptive responses that underlie reward systems with unrestricted intake of a highly palatable diet leading to an increase in the "reinforcing value of food" and weakened inhibitory control $(79,80)$. In addition, whereas ghrelin originally emerged as a gut-derived hormone involved in hunger and meal initiation and energy balance via effects on the hypothalamus, it also appears to have a role in reward-driven behaviors (81). This occurs via stimulation of the cholinergicdopaminergic reward pathway via inputs to the ventral tegmental area and in the mesolimbic pathway, a circuit that is known to communicate the hedonic and reinforcing facets of natural rewards such as high fat/high sugar food. However, changes in these reward systems and the potential for ghrelin-mediated alterations in reward behavior have yet to be defined in the context of early life programming. Given that exposure to obesogenic diets during early life can impact upon the neurocircuitry that is involved in behavioral motivation (79), studies in the setting of maternal obesity and offspring behavior are clearly warranted.

\section{INTERVENTIONS}

A range of animal models have allowed for investigation of a number of experimental intervention paradigms (including pharmacologic and nutritional) aimed at reversing or ameliorating the effects of early life programming. A number of animal studies have shown that some adverse metabolic outcomes, manifest due to adverse developmental programming, can be rescued by targeted interventions during early life (82). It is important, however, to recognize potential sex-specific responsiveness to interventions and there is not a "one size fits all." This was highlighted in the leptin intervention studies whereby sexually dimorphic responses to neonatal leptin treatment were reported $(83,84)$ and, importantly, early leptin treatment to male offspring of control pregnancies has the potential to induce an adverse metabolic phenotype in adulthood including hyperinsulinemia and increased adiposity (84). Further work also showed that the effects of neonatal leptin treatment on gene expression and
DNA methylation in the livers of adult offspring appeared to be directionally dependent on maternal nutritional status although neuroendocrine changes were not examined (85).

\section{Leptin}

Leptin has received significant attention as key mediator of developmental programming with alterations in the leptin in early life linked to an increased risk for obesity and metabolic dysregulation in later life (86). Leptin, under normal physiologic conditions, plays a critical role as a regulator of energy balance and acts centrally to control appetite and promote energy expenditure. Peripherally, it acts to reduce ectopic fat deposition, protect against lipotoxicity and to maintain glucose homeostasis.

Manipulations of leptin levels in early life in a range of model species utilizing leptin and/or leptin antagonists have highlighted a key role for leptin in the reprogramming of metabolic outcomes in offspring (86-89). During the "plastic" phase of early development (typically in rodents the first two weeks following birth), a number of studies have now clearly shown that maintenance of a critical leptin level is essential for the normal development of tissues and signaling pathways involved in metabolic control (86). As such, periods of relative hyper- or hypoleptinemia during this early developmental window may induce some of the metabolic adaptations which arise as a consequence of aberrant developmental programming. The mechanisms by which leptin influences the early developmental processes is still being defined and requires an understanding of the processes around the timing and amplitude of the early leptin surge, sex-specific effects, optimizing responsiveness to exogenous leptin treatment (i.e., critical windows), and a more in-depth understanding of leptins role in the development of the neurocircuitry involved in appetite control and energy homeostasis.

Most work to date using leptin as a programming "reversal agent" has been modeled in the rodent and there remains a paucity of evidence to support a primary role for leptin in the normal development and maturation of ARH wiring in higher model species (90). As an example, leptin levels are virtually undetectable in the NHP until the middle of the final trimester and parallel the late maturation of adipose tissue; this is evident even in fetal offspring from pregnancies characterized by obesity and hyperleptinemia (91). There may be a role for leptin in the refinement and branching networks of the ARH-NPY/AgRP projections closer to the time of parturition (92) but this has not been well characterized.

It must be noted however that, although leptin has received considerable attention as a key programming factor, work by Cottrell et al. has also provided evidence that programming of metabolic outcomes due to rapid catch-up growth per se does not require leptin signals (93). Using the $o b / o b$ leptin deficient mouse model, low birth weight followed by rapid catch-up growth in the preweaning period resulted in an increase in body weight in adulthood characterized by hyperphagia and increased adiposity. These results indicated that factors independent of leptin were involved in the programming of energy homeostasis in this model. Further, recent work by Sominsky et al. showed that changes in leptin availability during early life influences development of hypothalamic networks in the short term but is partly 
resolved by adulthood (94). In this study, neonatal overfeeding led to an acute resistance of hypothalamic neurons to exogenous leptin and an early increase in AgRP/NPY fiber number which was resolved in adulthood. Neonatal leptin antagonism did not reverse the excess body weight or hyperleptinemia in the group overfed as neonates, thus suggesting factors other than leptin may contribute to the offspring phenotype (94).

\section{Other Interventions}

Work by Steculorum et al. in the mouse has shown that the uridine-diphosphate (UDP) receptor (P2Y6) signaling in AgRP neurons is involved in the initiation of obesity-associated hyperphagia and systemic insulin resistance (95). Central injection of UDP acutely promoted feeding in diet-induced obese mice and that pharmacological blockade of CNS P2Y6 receptors reduced food intake. These data suggest that P2Y6 may be a potential pharmacologic target to restrict both feeding and systemic insulin resistance in obesity and that P2Y6 receptor antagonism may have efficacy in reversing central programming effects in offspring induced as a consequence of maternal obesity.

It is now well-established that changes in the availability of dietary methyl donors can impact on the gene expression patterns by affecting DNA methylation at regulatory regions, a likely mediator for reprogramming effects developmental plasticity (96). In the non-pregnant state, methyl donor supplementation can reduce hepatic steatosis and modify the DNA methylation profile of fatty acid synthase (FAS) in rats fed an obesogenic diet (97). Maternal methyl supplements have recently been shown to exert lasting protective effects in offspring of HF-fed dams with changes in DNA methylation profiles of peroxisome proliferator activate receptor- $\gamma$, FAS, leptin, and adiponectin (98). Similarly, supplementation with methyl donors (including choline, betaine, folic acid, and vitamin $\mathrm{B}_{12}$ ) during the lactation phase can prevent the elevations in plasma homocysteine in offspring induced by a $\mathrm{HF} /$ high sugar intake in rat dams (99) and was linked to altered regulation of the methionine-homocysteine cycle. However, these studies have focused on peripheral tissues and yet to report on potential changes in the hypothalamic regions of interest. Work in the mouse has shown that maternal HFD consumption can lead to global and gene-specific decreases in DNA methylation in the brains of offspring; effects which are ameliorated with maternal methyl donor supplements although in a sex-specific manner (100).

\section{DISCUSSION}

The neural circuitry involved in the regulation of appetite and energy homeostasis has been extensively studied over the last two decades. However, although the anatomy and functional plasticity of the BBB has been well characterized, the molecular mechanisms regulating the interface and integration of peripheral metabolic signals to the ARH still remain poorly defined (101). A consensus has emerged that early life exposure to a maternal obesogenic environment increases the risk of developing obesity and metabolic disorders in adulthood. However, there is less agreement on the mechanisms through which such risk may be conferred (4). Collectively, data to date reveal that leptin, in particular, and ghrelin play key roles in facilitating the normal development of hypothalamic neural circuits and suggest that normal expression of these factors during the fetal/neonatal period is key for lifelong metabolic regulation $(55,86,102)$. Although most work to date has been undertaken in the rodent where the early neonatal period is seen as the critical period for development of the neuroendocrine circuits, in many species the neuropeptide systems which control food intake develop and mature in utero, with large differences across the model species used (90).

Experimental animal data has provided some insights into mechanisms and critical windows; it also opens up more questions. As an example, how do alterations in maternal metabolic homeostasis alter the BBB interface in the ARH of offspring at birth? (101). Experimentally, further studies examining altered accessibility of circulating metabolic signals to ARH neurons at birth and/or the neonatal period during the period of neuronal plasticity to examine longer term impacts is also justified (101). Given that leptin, insulin, and ghrelin have all been shown to play a part in central reprogramming of neuronal wiring, does altering $\mathrm{BBB}$ access lead to development of central hormone resistance and disease in later life? Future work will also need to examine the transgenerational impacts of neuroendocrine programming following developmental exposures to a HFD through both maternal and paternal lineages (14). Although maternal HFD effects have been shown to extend through to the third generation (103), little is known re sex-specific programming effects and the central mechanisms involved. This raises a limitation of some experimental approaches whereby balanced experimental designs are not utilized thus precluding detailed examination of gene-environment interactions and further, sex-specific effects are often not examined. This is particularly true for intervention studies whereby it has evidenced shown that intervening in a replete environment may cause harm. An example of this is maternal taurine supplementation in a rat model of maternal obesity; supplementation to obese mothers decreased the high neonatal motility observed in this group but increased neonatal mortality in offspring of supplemented control dams, possibly due to the hypoglycemic effects of taurine (104). As detailed above, similar outcomes have also been reported for neonatal leptin treatment where offspring outcomes are directionally dependent on maternal nutritional background and offspring sex (83-85).

Circulating hormones integrate and impact upon multiple components of hypothalamic development and therefore play a key role in the coordination of neural circuit formation and normalization of neuroendocrine signaling pathways. It is likely therefore that deficiencies in any of these factors during sensitive periods of developmental plasticity can lead to lasting structural and functional consequences. As detailed above, in model species that give birth to mature young (e.g., NHP, sheep) maximal brain growth and a large proportion of neuroendocrine maturation takes occurs in fetal life $(105,106)$. Rodents are born immature and thus hypothalamic maturation is initiated prenatally but not finalized until the second week of postnatal life (107). Maternal lactational capacity therefore is a primary determinant of nutrient supply in the rodent rather than dependence upon the nutrient 
transfer capacity of the placenta. Alterations in the composition of milk during the suckling phase therefore have the potential to exert marked effects on hypothalamic maturation that can be mediated through changes in milk hormone concentrations. For example, rat neonates born to obese mothers exhibit a precocious surge in circulating insulin paralleled by abnormalities in the neonatal leptin surge (amplified and prolonged) with concomitant elevations in leptin mRNA expression in visceral adipose tissue (19). It was hypothesized that extended release of high leptin concentrations by neonatal rats born to obese mothers resulted in leptin resistance and permanent effects on hypothalamic function involving the ARH and PVN and therefore may underlie the later hyperphagia and obesity observed in these animals. These effects are likely mediated in part by alterations in milk composition with a maternal HFD in the rat shown to be associated with increased milk protein and lipid concentrations $(108,109)$. These changes in compositional profile result in neonatal overnutrition and can result in thyroid and adrenal dysfunction and increased adiposity in male offspring as early as weaning (110). However, despite the well-characterized developmental differences across the range of model species used in programming studies, both precocial and altricial species appear to exhibit several potential commonalities in programming mechanisms that result in the altered adult phenotype, particularly as regards alterations in hypothalamic wiring.

Although epigenetic mechanisms can mediate the effects of adverse maternal obesity during pregnancy/lactation and offspring developmental malprogramming, it has also been suggested that increases in accessibility of ARH neurons to peripheral metabolic signals could arise via processes similar to that seen with the adaptive responses to fasting (111). The BBB restricts delivery of circulating factors that transfer metabolic information to the neural networks that regulate energy homeostasis. During fasting, the decrease in blood glucose levels alters the structural organization of the blood-hypothalamus barrier and results in the improved access of metabolic substrates to the ARH (111). These data that glucose plays a role in the control of blood-hypothalamic exchange through a vascular endothelial growth factor (VEGF)-dependent mechanism and demonstrated a role for tanycytes and associated permeable microvessels in the adaptive metabolic response to fasting. This mechanism could also translate to strategies for intervention-treatment of offspring of obese mothers with VEGF receptor inhibitors could prevent the deleterious effects of maternal obesity by altering capillary fenestration of the median eminence branches extending to the ARH.

\section{REFERENCES}

1. Reddon H, Gueant JL, Meyre D. The importance of gene-environment interactions in human obesity. Clin Sci (Lond) (2016) 130:1571-97. doi:10.1042/ CS20160221

2. Huang T, Hu FB. Gene-environment interactions and obesity: recent developments and future directions. BMC Med Genomics (2015) 8:S2. doi:10.1186/ 1755-8794-8-S1-S2

3. Levin BE. Epigenetic influences on food intake and physical activity level: review of animal studies. Obesity (Silver Spring) (2008) 16:S51-4. doi:10.1038/ oby. 2008.518
Wherever possible, animal models of maternal obesity should be designed based on the principals recently developed via the Animals in Research: Reporting In Vivo Experiments guidelines (112). This includes detailed reporting of the species/strain/sex of the animals used, balanced experimental design, detailed dietary composition details and implementation of processes around randomization and blinding to reduce experimental bias in animal selection. Such approaches will allow increased relevance of studies undertaken in the setting of maternal obesity and increase translative capacity to the human setting. As above, many studies to date have not examined the impact of sexual dimorphic responses to different programming stimuli and these differences need to be acknowledged as they inform on the mechanistic underpinnings of how divergent phenotypes emerge. Adoption of a life course approach to examine phenotype development allows early identification of related risk markers $(113,114)$, with the potential that nutritional, pharmacological and lifestyle (e.g., exercise) interventions may aid in the amelioration of noncommunicable diseases, particularly in those developing societies that are in the process of nutritional transition. The different animal models utilized will have different utility as regards translation to the human condition. It is noteworthy however, that despite the differences in timing of neurodevelopmental processes across the models species used and the differences in dietary compositions and duration of exposures, the phenotypes reported often mirror those seen in the clinical setting of the metabolic syndrome. This speaks to the validity of the range of animal models currently used to investigate the neuroendocrine mechanisms underpinning developmental programming. As perinatal manipulations can permanently alter the systems involved in appetite control and energy homeostasis, there is an urgent need to identify the key factors responsible as a means of stemming the worldwide obesity epidemic via effective targeted intervention strategies during critical early periods of developmental plasticity.

\section{AUTHOR CONTRIBUTIONS}

MV wrote the first draft of the review and then was further revised and updated by SS and CR.

\section{FUNDING}

The authors acknowledge funding support from the Health Research Council of New Zealand, the Faculty Research Development Fund (University of Auckland), and the Auckland Medical Research Foundation.

4. Walker CD. Development, brain plasticity and reward: early high-fat diet exposure confers vulnerability to obesity-view from the chair. Int J Obes Suppl (2012) 2:S3-6. doi:10.1038/ijosup.2012.14

5. Kim DW, Glendining KA, Grattan DR, Jasoni CL. Maternal obesity in the mouse compromises the blood-brain barrier in the arcuate nucleus of offspring. Endocrinology (2016) 157:2229-42. doi:10.1210/en.2016-1014

6. Penfold NC, Ozanne SE. Developmental programming by maternal obesity in 2015: outcomes, mechanisms, and potential interventions. Horm Behav (2015) 76:143-52. doi:10.1016/j.yhbeh.2015.06.015

7. Bouret SG, Draper SJ, Simerly RB. Trophic action of leptin on hypothalamic neurons that regulate feeding. Science (2004) 304:108-10. doi:10.1126/science.1095004 
8. Bouret SG, Simerly RB. Development of leptin-sensitive circuits. J Neuroendocrinol (2007) 19:575-82. doi:10.1111/j.1365-2826.2007.01563.x

9. Steculorum SM, Bouret SG. Maternal diabetes compromises the organization of hypothalamic feeding circuits and impairs leptin sensitivity in offspring. Endocrinology (2011) 152:4171-9. doi:10.1210/en.2011-1279

10. de la Monte SM, Wands JR. Review of insulin and insulin-like growth factor expression, signaling, and malfunction in the central nervous system: relevance to Alzheimer's disease. J Alzheimer Dis (2005) 7:45-61. doi:10.3233/ JAD-2005-7106

11. Blazquez E, Velazquez E, Hurtado-Carneiro V, Ruiz-Albusac JM. Insulin in the brain: its pathophysiological implications for states related with central insulin resistance, type 2 diabetes and Alzheimer's disease. Front Endocrinol (2014) 5:161. doi:10.3389/fendo.2014.00161

12. Kullmann S, Heni M, Hallschmid M, Fritsche A, Preissl H, Haring HU. Brain insulin resistance at the crossroads of metabolic and cognitive disorders in humans. Physiol Rev (2016) 96:1169-209. doi:10.1152/physrev.00032.2015

13. Jasoni CL, Sanders TR, Kim DW. Do all roads lead to Rome? The role of neuro-immune interactions before birth in the programming of offspring obesity. Front Neurosci (2014) 8:455. doi:10.3389/fnins.2014.00455

14. Aiken CE, Ozanne SE. Transgenerational developmental programming. Hum Reprod Update (2014) 20:63-75. doi:10.1093/humupd/dmt043

15. Sullivan EL, Smith MS, Grove KL. Perinatal exposure to high-fat diet programs energy balance, metabolism and behavior in adulthood. Neuroendocrinology (2011) 93:1-8. doi:10.1159/000322038

16. Grove KL, Grayson BE, Glavas MM, Xiao XQ, Smith MS. Development of metabolic systems. Physiol Behav (2005) 86:646-60. doi:10.1016/j.physbeh. 2005.08.063

17. Baquero AF, de Solis AJ, Lindsley SR, Kirigiti MA, Smith MS, Cowley MA, et al. Developmental switch of leptin signaling in arcuate nucleus neurons. J Neurosci (2014) 34:9982-94. doi:10.1523/JNEUROSCI.0933-14.2014

18. Howie GJ, Sloboda DM, Kamal T, Vickers MH. Maternal nutritional history predicts obesity in adult offspring independent of postnatal diet. J Physiol (2009) 587:905-15. doi:10.1113/jphysiol.2008.163477

19. Kirk SL, Samuelsson AM, Argenton M, Dhonye H, Kalamatianos T, Poston L, et al. Maternal obesity induced by diet in rats permanently influences central processes regulating food intake in offspring. PLoS One (2009) 4:e5870. doi:10.1371/journal.pone.0005870

20. Vogt MC, Paeger L, Hess S, Steculorum SM, Awazawa M, Hampel B, et al. Neonatal insulin action impairs hypothalamic neurocircuit formation in response to maternal high-fat feeding. Cell (2014) 156:495-509. doi:10.1016/ j.cell.2014.01.008

21. Chen H, Simar D, Lambert K, Mercier J, Morris MJ. Maternal and postnatal overnutrition differentially impact appetite regulators and fuel metabolism. Endocrinology (2008) 149:5348-56. doi:10.1210/en.2008-0582

22. Rajia S, Chen H, Morris MJ. Maternal overnutrition impacts offspring adiposity and brain appetite markers-modulation by postweaning diet. J Neuroendocrinol (2010) 22:905-14. doi:10.1111/j.1365-2826.2010.02005.x

23. Chen H, Simar D, Morris MJ. Hypothalamic neuroendocrine circuitry is programmed by maternal obesity: interaction with postnatal nutritional environment. PLoS One (2009) 4:e6259. doi:10.1371/journal.pone.0006259

24. Chang GQ, Gaysinskaya V, Karatayev O, Leibowitz SF. Maternal high-fat diet and fetal programming: increased proliferation of hypothalamic peptideproducing neurons that increase risk for overeating and obesity. J Neurosci (2008) 28:12107-19. doi:10.1523/JNEUROSCI.2642-08.2008

25. Kim DW, Glendining KA, Grattan DR, Jasoni CL. Maternal obesity leads to increased proliferation and numbers of astrocytes in the developing fetal and neonatal mouse hypothalamus. Int J Dev Neurosci (2016) 53:18-25. doi:10.1016/j.ijdevneu.2016.06.005

26. Hatanaka Y, Kabuta T, Wada K. Disturbance in maternal environment leads to abnormal synaptic instability during neuronal circuitry development. Front Neurosci (2017) 11:35. doi:10.3389/fnins.2017.00035

27. Hatanaka Y, Wada K, Kabuta T. Maternal high-fat diet leads to persistent synaptic instability in mouse offspring via oxidative stress during lactation. Neurochem Int (2016) 97:99-108. doi:10.1016/j.neuint.2016.03.008

28. Kim JG, Suyama S, Koch M, Jin S, Argente-Arizon P, Argente J, et al. Leptin signaling in astrocytes regulates hypothalamic neuronal circuits and feeding. Nat Neurosci (2014) 17:908-10. doi:10.1038/nn.3725

29. Desai M, Han G, Ross MG. Programmed hyperphagia in offspring of obese dams: altered expression of hypothalamic nutrient sensors, neurogenic factors and epigenetic modulators. Appetite (2016) 99:193-9. doi:10.1016/j. appet.2016.01.023

30. Samuelsson AS, Mullier A, Maicas N, Oosterhuis NR, Eun Bae S, Novoselova TV, et al. Central role for melanocortin-4 receptors in offspring hypertension arising from maternal obesity. Proc Natl Acad Sci U S A (2016) 113:12298-303. doi:10.1073/pnas.1607464113

31. Sampey BP, Vanhoose AM, Winfield HM, Freemerman AJ, Muehlbauer MJ, Fueger PT, et al. Cafeteria diet is a robust model of human metabolic syndrome with liver and adipose inflammation: comparison to high-fat diet. Obesity (Silver Spring) (2011) 19:1109-17. doi:10.1038/oby.2011.18

32. Ong ZY, Muhlhausler BS. Maternal "junk-food" feeding of rat dams alters food choices and development of the mesolimbic reward pathway in the offspring. FASEB J (2011) 25:2167-79. doi:10.1096/fi.10-178392

33. Dyer JS, Rosenfeld CR. Metabolic imprinting by prenatal, perinatal, and postnatal overnutrition: a review. Semin Reprod Med (2011) 29:266-76. doi :10.1055/s-0031-1275521

34. Chen H, Morris MJ. Differential responses of orexigenic neuropeptides to fasting in offspring of obese mothers. Obesity (Silver Spring) (2009) 17:1356-62. doi:10.1038/oby.2009.56

35. Wang H, Storlien LH, Huang XF. Effects of dietary fat types on body fatness, leptin, and ARC leptin receptor, NPY, and AgRP mRNA expression. Am J Physiol Endocrinol Metab (2002) 282:E1352-9. doi:10.1152/ajpendo. 00230.2001

36. Long NM, Ford SP, Nathanielsz PW. Maternal obesity eliminates the neonatal lamb plasma leptin peak. J Physiol (2011) 589:1455-62. doi:10.1113/ jphysiol.2010.201681

37. Muhlhausler BS, McMillen IC, Rouzaud G, Findlay PA, Marrocco EM, Rhind SM, et al. Appetite regulatory neuropeptides are expressed in the sheep hypothalamus before birth. J Neuroendocrinol (2004) 16:502-7. doi:10.1111/j.1365-2826.2004.01197.x

38. Long NM, Rule DC, Zhu MJ, Nathanielsz PW, Ford SP. Maternal obesity upregulates fatty acid and glucose transporters and increases expression of enzymes mediating fatty acid biosynthesis in fetal adipose tissue depots. J Anim Sci (2012) 90:2201-10. doi:10.2527/jas.2011-4343

39. Nicholas LM, Rattanatray L, Morrison JL, Kleemann DO, Walker SK, Zhang S, et al. Maternal obesity or weight loss around conception impacts hepatic fatty acid metabolism in the offspring. Obesity (Silver Spring) (2014) 22:1685-93. doi:10.1002/oby.20752

40. Long NM, Rule DC, Tuersunjiang N, Nathanielsz PW, Ford SP. Maternal obesity in sheep increases fatty acid synthesis, upregulates nutrient transporters, and increases adiposity in adult male offspring after a feeding challenge. PLoS One (2015) 10:e0122152. doi:10.1371/journal.pone.0122152

41. McMillen IC, Adam CL, Muhlhausler BS. Early origins of obesity: programming the appetite regulatory system. J Physiol (2005) 565:9-17. doi:10.1113/ jphysiol.2004.081992

42. Muhlhausler BS, Adam CL, Findlay PA, Duffield JA, McMillen IC. Increased maternal nutrition alters development of the appetite-regulating network in the brain. FASEB J (2006) 20:1257-9. doi:10.1096/fj.05-5241fje

43. Muhlhausler BS, Adam CL, Marrocco EM, Findlay PA, Roberts CT, McFarlane JR, et al. Impact of glucose infusion on the structural and functional characteristics of adipose tissue and on hypothalamic gene expression for appetite regulatory neuropeptides in the sheep fetus during late gestation. J Physiol (2005) 565:185-95. doi:10.1113/jphysiol.2004.079079

44. Attig L, Djiane J, Gertler A, Rampin O, Larcher T, Boukthir S, et al. Study of hypothalamic leptin receptor expression in low-birth-weight piglets and effects of leptin supplementation on neonatal growth and development. Am J Physiol Endocrinol Metab (2008) 295:E1117-25. doi:10.1152/ajpendo. 90542.2008

45. Val-Laillet D, Besson M, Guerin S, Coquery N, Randuineau G, Kanzari A, et al. A maternal Western diet during gestation and lactation modifies offspring's microbiota activity, blood lipid levels, cognitive responses, and hippocampal neurogenesis in Yucatan pigs. FASEB J (2017) 31:2037-49. doi:10.1096/ f. $201601015 R$

46. Sanguinetti E, Liistro T, Mainardi M, Pardini S, Salvadori PA, Vannucci A, et al. Maternal high-fat feeding leads to alterations of brain glucose metabolism in the offspring: positron emission tomography study in a porcine model. Diabetologia (2016) 59:813-21. doi:10.1007/s00125-015-3848-5

47. Prior LJ, Davern PJ, Burke SL, Lim K, Armitage JA, Head GA. Exposure to a high-fat diet during development alters leptin and ghrelin sensitivity and 
elevates renal sympathetic nerve activity and arterial pressure in rabbits. Hypertension (2014) 63:338-45. doi:10.1161/HYPERTENSIONAHA.113.02498

48. Prior LJ, Eikelis N, Armitage JA, Davern PJ, Burke SL, Montani JP, et al. Exposure to a high-fat diet alters leptin sensitivity and elevates renal sympathetic nerve activity and arterial pressure in rabbits. Hypertension (2010) 55:862-8. doi:10.1161/HYPERTENSIONAHA.109.141119

49. Lim JK, Burke S, Head G. OS 29-01 trans-generational programming of obesity related hypertension: amplifiaction of leptin signalling pathway. J Hypertens (2016) 34:e253. doi:10.1097/01.hjh.0000500573.17791.04

50. Sullivan EL, Grayson B, Takahashi D, Robertson N, Maier A, Bethea CL, et al. Chronic consumption of a high-fat diet during pregnancy causes perturbations in the serotonergic system and increased anxiety-like behavior in nonhuman primate offspring. J Neurosci (2010) 30:3826-30. doi:10.1523/ JNEUROSCI.5560-09.2010

51. Grayson BE, Levasseur PR, Williams SM, Smith MS, Marks DL, Grove KL. Changes in melanocortin expression and inflammatory pathways in fetal offspring of nonhuman primates fed a high-fat diet. Endocrinology (2010) 151:1622-32. doi:10.1210/en.2009-1019

52. Rivera HM, Kievit P, Kirigiti MA, Bauman LA, Baquero K, Blundell P, et al. Maternal high-fat diet and obesity impact palatable food intake and dopamine signaling in nonhuman primate offspring. Obesity (Silver Spring) (2015) 23:2157-64. doi:10.1002/oby.21306

53. Prinz P, Stengel A. Control of food intake by gastrointestinal peptides: mechanisms of action and possible modulation in the treatment of obesity. J Neurogastroenterol Motil (2017) 23:180-96. doi:10.5056/jnm16194

54. Hewson AK, Tung LY, Connell DW, Tookman L, Dickson SL. The rat arcuate nucleus integrates peripheral signals provided by leptin, insulin, and a ghrelin mimetic. Diabetes (2002) 51:3412-9. doi:10.2337/diabetes.51.12.3412

55. Steculorum SM, Collden G, Coupe B, Croizier S, Lockie S, Andrews ZB, et al. Neonatal ghrelin programs development of hypothalamic feeding circuits. J Clin Invest (2015) 125:846-58. doi:10.1172/JCI73688

56. Schele E, Bake T, Rabasa C, Dickson SL. Centrally administered ghrelin acutely influences food choice in rodents. PLoS One (2016) 11:e0149456. doi:10.1371/ journal.pone. 0149456

57. Briggs DI, Enriori PJ, Lemus MB, Cowley MA, Andrews ZB. Diet-induced obesity causes ghrelin resistance in arcuate NPY/AgRP neurons. Endocrinology (2010) 151:4745-55. doi:10.1210/en.2010-0556

58. Collden G, Balland E, Parkash J, Caron E, Langlet F, Prevot V, et al. Neonatal overnutrition causes early alterations in the central response to peripheral ghrelin. Mol Metab (2015) 4:15-24. doi:10.1016/j.molmet.2014.10.003

59. Sominsky L, Ziko I, Nguyen TX, Andrews ZB, Spencer SJ. Early life disruption to the ghrelin system with over-eating is resolved in adulthood in male rats. Neuropharmacology (2017) 113:21-30. doi:10.1016/j.neuropharm. 2016.09.023

60. Sominsky L, Ziko I, Spencer SJ. Neonatal overfeeding disrupts pituitary ghrelin signalling in female rats long-term; Implications for the stress response. PLoS One (2017) 12:e173498. doi:10.1371/journal.pone.0173498

61. Godfrey KM, Sheppard A, Gluckman PD, Lillycrop KA, Burdge GC, McLean C, et al. Epigenetic gene promoter methylation at birth is associated with child's later adiposity. Diabetes (2011) 60:1528-34. doi:10.2337/db10-0979

62. Liu HW, Mahmood S, Srinivasan M, Smiraglia DJ, Patel MS. Developmental programming in skeletal muscle in response to overnourishment in the immediate postnatal life in rats. J Nutr Biochem (2013) 24:1859-69. doi:10.1016/ j.jnutbio.2013.05.002

63. Plagemann A, Roepke K, Harder T, Brunn M, Harder A, Wittrock-Staar M, et al. Epigenetic malprogramming of the insulin receptor promoter due to developmental overfeeding. J Perinat Med (2010) 38:393-400. doi:10.1515/ JPM.2010.051

64. Plagemann A, Harder T, Brunn M, Harder A, Roepke K, Wittrock-Staar M, et al. Hypothalamic proopiomelanocortin promoter methylation becomes altered by early overfeeding: an epigenetic model of obesity and the metabolic syndrome. J Physiol (2009) 587:4963-76. doi:10.1113/jphysiol. 2009.176156

65. Marco A, Kisliouk T, Tabachnik T, Meiri N, Weller A. Overweight and CpG methylation of the Pomc promoter in offspring of high-fat-diet-fed dams are not "reprogrammed" by regular chow diet in rats. FASEB J (2014) 28:4148-57. doi:10.1096/f.14-255620

66. Dudley KJ, Sloboda DM, Connor KL, Beltrand J, Vickers MH. Offspring of mothers fed a high fat diet display hepatic cell cycle inhibition and associated changes in gene expression and DNA methylation. PLoS One (2011) 6:e21662. doi:10.1371/journal.pone. 0021662

67. Chernigovskaya EV, Taranukhin AG, Glazova MV, Yamova LA, Fedorov LM. Apoptotic signaling proteins: possible participation in the regulation of vasopressin and catecholamines biosynthesis in the hypothalamus. Histochem Cell Biol (2005) 124:523-33. doi:10.1007/s00418-005-0016-x

68. Guarnieri DJ, Brayton CE, Richards SM, Maldonado-Aviles J, Trinko JR, Nelson J, et al. Gene profiling reveals a role for stress hormones in the molecular and behavioral response to food restriction. Biol Psychiatry (2012) 71:358-65. doi:10.1016/j.biopsych.2011.06.028

69. Ring RH, Valo Z, Gao C, Barish ME, Singer-Sam J. The Cdkn1a gene (p21Waf1/ Cip1) is an inflammatory response gene in the mouse central nervous system. Neurosci Lett (2003) 350:73-6. doi:10.1016/S0304-3940(03)00883-8

70. Vucetic Z, Kimmel J, Totoki K, Hollenbeck E, Reyes TM. Maternal high-fat diet alters methylation and gene expression of dopamine and opioid-related genes. Endocrinology (2010) 151:4756-64. doi:10.1210/en.2010-0505

71. Strakovsky RS, Zhang X, Zhou D, Pan YX. The regulation of hepatic Pon 1 by a maternal high-fat diet is gender specific and may occur through promoter histone modifications in neonatal rats. J Nutr Biochem (2014) 25:170-6. doi:10.1016/j.jnutbio.2013.09.016

72. Suter MA, Chen A, Burdine MS, Choudhury M, Harris RA, Lane RH, et al. A maternal high-fat diet modulates fetal SIRT1 histone and protein deacetylase activity in nonhuman primates. FASEB J (2012) 26:5106-14. doi:10.1096/ f.12-212878

73. Nguyen LT, Chen H, Pollock CA, Saad S. Sirtuins-mediators of maternal obesity-induced complications in offspring? FASEB J (2016) 30:1383-90. doi:10.1096/f.15-280743

74. Hawkins PG, Morris KV. RNA and transcriptional modulation of gene expression. Cell Cycle (2008) 7:602-7. doi:10.4161/cc.7.5.5522

75. Yan X, Huang Y, Zhao JX, Rogers CJ, Zhu MJ, Ford SP, et al. Maternal obesity downregulates microRNA let-7g expression, a possible mechanism for enhanced adipogenesis during ovine fetal skeletal muscle development. Int J Obes (Lond) (2013) 37:568-75. doi:10.1038/ijo.2012.69

76. Kim JD, Toda C, Ramirez CM, Fernandez-Hernando C, Diano S. Hypothalamic ventromedial Lin28a enhances glucose metabolism in diet-induced obesity. Diabetes (2017) 66:2102-11. doi:10.2337/db16-1558

77. Grissom NM, Lyde R, Christ L, Sasson IE, Carlin J, Vitins AP, et al. Obesity at conception programs the opioid system in the offspring brain. Neuropsychopharmacology (2014) 39:801-10. doi:10.1038/npp.2013.193

78. Hu Z, Cooper M, Crockett DP, Zhou R. Differentiation of the midbrain dopaminergic pathways during mouse development. J Comp Neurol (2004) 476:301-11. doi:10.1002/cne.20230

79. Reichelt AC, Morris MJ, Westbrook RF. Cafeteria diet impairs expression of sensory-specific satiety and stimulus-outcome learning. Front Psychol (2014) 5:852. doi:10.3389/fpsyg.2014.00852

80. Gugusheff JR, Ong ZY, Muhlhausler BS. Naloxone treatment alters gene expression in the mesolimbic reward system in 'junk food' exposed offspring in a sex-specific manner but does not affect food preferences in adulthood. Physiol Behav (2014) 133:14-21. doi:10.1016/j.physbeh.2014.04.007

81. Dickson SL, Egecioglu E, Landgren S, Skibicka KP, Engel JA, Jerlhag E. The role of the central ghrelin system in reward from food and chemical drugs. Mol Cell Endocrinol (2011) 340:80-7. doi:10.1016/j.mce.2011.02.017

82. Vickers MH, Sloboda DM. Strategies for reversing the effects of metabolic disorders induced as a consequence of developmental programming. Front Physiol (2012) 3:242. doi:10.3389/fphys.2012.00242

83. Vickers MH, Gluckman PD, Coveny AH, Hofman PL, Cutfield WS, Gertler A, et al. Neonatal leptin treatment reverses developmental programming. Endocrinology (2005) 146:4211-6. doi:10.1210/en.2005-0581

84. Vickers MH, Gluckman PD, Coveny AH, Hofman PL, Cutfield WS, Gertler A, et al. The effect of neonatal leptin treatment on postnatal weight gain in male rats is dependent on maternal nutritional status during pregnancy. Endocrinology (2008) 149:1906-13. doi:10.1210/en.2007-0981

85. Gluckman PD, Lillycrop KA, Vickers MH, Pleasants AB, Phillips ES, Beedle AS, et al. Metabolic plasticity during mammalian development is directionally dependent on early nutritional status. Proc Natl Acad Sci U S A (2007) 104: 12796-800. doi:10.1073/pnas.0705667104

86. Vickers MH, Sloboda DM. Leptin as mediator of the effects of developmental programming. Best Pract Res Clin Endocrinol Metab (2012) 26:677-87. doi:10.1016/j.beem.2012.03.005 
87. Vickers MH. Developmental programming and adult obesity: the role of leptin. Curr Opin Endocrinol Diabetes Obes (2007) 14:17-22. doi:10.1097/ MED.0b013e328013da48

88. Attig L, Solomon G, Ferezou J, Abdennebi-Najar L, Taouis M, Gertler A, et al. Early postnatal leptin blockage leads to a long-term leptin resistance and susceptibility to diet-induced obesity in rats. Int J Obes (Lond) (2008) 32:1153-60. doi:10.1038/ijo.2008.39

89. Beltrand J, Sloboda D, Connor K, Truong M, Vickers M. The effect of neonatal leptin antagonism in male rat offspring is dependent upon the interaction between prior maternal nutritional status and post-weaning diet. J Nutr Metab (2012) 2012:296935. doi:10.1155/2012/296935

90. Grayson BE, Kievit P, Smith MS, Grove KL. Critical determinants of hypothalamic appetitive neuropeptide development and expression: species considerations. Front Neuroendocrinol (2010) 31:16-31. doi:10.1016/j.yfrne. 2009.10.001

91. McCurdy CE, Bishop JM, Williams SM, Grayson BE, Smith MS, Friedman JE, et al. Maternal high-fat diet triggers lipotoxicity in the fetal livers of nonhuman primates. J Clin Invest (2009) 119:323-35. doi:10.1172/JCI32661

92. Horvath TL. Synaptic plasticity mediating leptin's effect on metabolism. Prog Brain Res (2006) 153:47-55. doi:10.1016/S0079-6123(06)53002-X

93. Cottrell EC, Martin-Gronert MS, Fernandez-Twinn DS, Luan J, Berends LM, Ozanne SE. Leptin-independent programming of adult body weight and adiposity in mice. Endocrinology (2011) 152:476-82. doi:10.1210/en.2010-0911

94. Sominsky L, Ziko I, Nguyen TX, Quach J, Spencer SJ. Hypothalamic effects of neonatal diet: reversible and only partially leptin-dependent. J Endocrinol (2017) 234(1):41-56. doi:10.1530/JOE-16-0631

95. Steculorum SM, Timper K, Engstrom Ruud L, Evers N, Paeger L, Bremser S, et al. Inhibition of P2Y6 signaling in AgRP neurons reduces food intake and improves systemic insulin sensitivity in obesity. Cell Rep (2017) 18:1587-97. doi:10.1016/j.celrep.2017.01.047

96. Navarro E, Funtikova AN, Fito M, Schroder H. Prenatal nutrition and the risk of adult obesity: long-term effects of nutrition on epigenetic mechanisms regulating gene expression. J Nutr Biochem (2017) 39:1-14. doi:10.1016/j. jnutbio.2016.03.012

97. Cordero P, Gomez-Uriz AM, Campion J, Milagro FI, Martinez JA. Dietary supplementation with methyl donors reduces fatty liver and modifies the fatty acid synthase DNA methylation profile in rats fed an obesogenic diet. Genes Nutr (2013) 8:105-13. doi:10.1007/s12263-012-0300-z

98. Jiao F, Yan X, Yu Y, Zhu X, Ma Y, Yue Z, et al. Protective effects of maternal methyl donor supplementation on adult offspring of high fat diet-fed dams. J Nutr Biochem (2016) 34:42-51. doi:10.1016/j.jnutbio.2016.04.005

99. Cordero P, Milagro FI, Campion J, Martinez JA. Maternal methyl donors supplementation during lactation prevents the hyperhomocysteinemia induced by a high-fat-sucrose intake by dams. Int J Mol Sci (2013) 14:24422-37. doi:10.3390/ijms141224422

100. Carlin J, George R, Reyes TM. Methyl donor supplementation blocks the adverse effects of maternal high fat diet on offspring physiology. PLoS One (2013) 8:e63549. doi:10.1371/journal.pone.0063549

101. Duquenne M, Dehouck B, Prevot V. Programming the brain from the womb: maternal obesity perturbs the hypothalamic blood-brain barrier. Endocrinology (2016) 157:2201-3. doi:10.1210/en.2016-1262

102. Frago LM, Chowen JA. Hypothalamic leptin and ghrelin signaling as targets for improvement in metabolic control. Curr Pharm Des (2015) 21:3596-605. doi:10.2174/1381612821666150710145428
103. Dunn GA, Bale TL. Maternal high-fat diet effects on third-generation female body size via the paternal lineage. Endocrinology (2011) 152:2228-36. doi:10.1210/en.2010-1461

104. Li M, Reynolds CM, Sloboda DM, Gray C, Vickers MH. Effects of taurine supplementation on hepatic markers of inflammation and lipid metabolism in mothers and offspring in the setting of maternal obesity. PLoS One (2013) 8:e76961. doi:10.1371/journal.pone.0076961

105. Ojha S, Fainberg HP, Sebert S, Budge H, Symonds ME. Maternal health and eating habits: metabolic consequences and impact on child health. Trends Mol Med (2015) 21:126-33. doi:10.1016/j.molmed.2014.12.005

106. Kapoor A, Petropoulos S, Matthews SG. Fetal programming of hypothalamic-pituitary-adrenal (HPA) axis function and behavior by synthetic glucocorticoids. Brain Res Rev (2008) 57:586-95. doi:10.1016/j. brainresrev.2007.06.013

107. Bouret SG, Simerly RB. Developmental programming of hypothalamic feeding circuits. Clin Genet (2006) 70:295-301. doi:10.1111/j.1399-0004. 2006.00684.x

108. Purcell RH, Sun B, Pass LL, Power ML, Moran TH, Tamashiro KL. Maternal stress and high-fat diet effect on maternal behavior, milk composition, and pup ingestive behavior. Physiol Behav (2011) 104:474-9. doi:10.1016/j. physbeh.2011.05.012

109. Loh TC, Foo HL, Abdul Wahab Z, Tan BK. Effects of feeding fat during pregnancy and lactation on growth performance, milk composition and very low density lipoprotein composition in rats. Malays J Nutr (2002) 8:125-35.

110. Franco JG, Fernandes TP, Rocha CP, Calvino C, Pazos-Moura CC, Lisboa PC, et al. Maternal high-fat diet induces obesity and adrenal and thyroid dysfunction in male rat offspring at weaning. J Physiol (2012) 590:5503-18. doi:10.1113/jphysiol.2012.240655

111. Langlet F, Levin BE, Luquet S, Mazzone M, Messina A, Dunn-Meynell AA, et al. Tanycytic VEGF-A boosts blood-hypothalamus barrier plasticity and access of metabolic signals to the arcuate nucleus in response to fasting. Cell Metab (2013) 17:607-17. doi:10.1016/j.cmet.2013.03.004

112. Kilkenny C, Browne WJ, Cuthill IC, Emerson M, Altman DG. Improving bioscience research reporting: the ARRIVE guidelines for reporting animal research. PLoS Biol (2010) 8:e1000412. doi:10.1371/journal.pbio.1000412

113. Hanson MA, Cooper C, Aihie Sayer A, Eendebak RJ, Clough GF, Beard JR. Developmental aspects of a life course approach to healthy ageing. J Physiol (2016) 594:2147-60. doi:10.1113/JP270579

114. Godfrey KM, Gluckman PD, Hanson MA. Developmental origins of metabolic disease: life course and intergenerational perspectives. Trends Endocrinol Metab (2010) 21:199-205. doi:10.1016/j.tem.2009.12.008

Conflict of Interest Statement: The authors declare that the research was conducted in the absence of any commercial or financial relationships that could be construed as a potential conflict of interest.

The reviewer LD and handling editor declared their shared affiliation.

Copyright (๑) 2017 Reynolds, Segovia and Vickers. This is an open-access article distributed under the terms of the Creative Commons Attribution License (CC BY). The use, distribution or reproduction in other forums is permitted, provided the original author(s) or licensor are credited and that the original publication in this journal is cited, in accordance with accepted academic practice. No use, distribution or reproduction is permitted which does not comply with these terms. 